\title{
Aboodh Homotopy Perturbation Method of Solving Burgers Equation
}

\author{
Oludapo Omotola Olubanwo ${ }^{1 *}$, Olutunde Samuel Odetunde ${ }^{1}$, Adetoro Temitope Talabi ${ }^{1}$ \\ ${ }^{1}$ Department of Mathematical Sciences, Olabisi Onabanjo University \\ Ago Iwoye, Nigeria \\ ${ }^{2}$ Department of Physics, Olabisi Onabanjo University \\ Ago Iwoye, Nigeria \\ *Corresponding author's email: olubanwo.oludapo@oouagoiwoye.edu.ng
}

\begin{abstract}
ABSRACT--- In this paper, we present a reliable combination of Aboodh Transform and Homotopy perturbation method to determine the exact solution of one dimensional Burgers equation which is a nonlinear partial differential equation. Some cases of one dimensional nonlinear partial differential equations are considered to illustrate the capability and reliability of Aboodh Homotopy perturbation method. We have compared the analytical solution obtained with the available Laplace decomposition method. The solution which is found to be exactly the same. The result revealed that the combination of the Aboodh Transform and homotopy perturbation method is quite capable, practically well appropriate for use in such problems. The method is tested on three examples and results show that the new method is more effective and convenient to use and high accuracy of it is evident.
\end{abstract}

Keywords-- Aboodh Transform, Homotopy Perturbation method, Burgers equation, Nonlinear Partial differential equation.

\section{INTRODUCTION}

Burgers' equation is obtained as a result of combining nonlinear wave motion with linear diffusion and is the simplest model for analyzing combined effect of nonlinear advection and diffusion. Nonlinear phenomena play a significant role in applied mathematics and physics. It is somehow difficult to obtain the exact solution for these problems. Recently, an increasing interest of researchers has been devoted to the analytical techniques for solving nonlinear problems. Several methods have been developed by many researchers to solve both linear and nonlinear partial differential equations; such as Backlund transformation method [1], Hirota's bilinear method [2], Variational iteration method [3], [4], Adomian Decomposition Method [5]. Homotopy Analysis Method [6], [7], [8], [9], The homotopy perturbation method (HPM) [10], [11], [12], [13].

In this study we use coupling of new integral transform "Aboodh transform" and homotopy perturbation method. Aboodh transform is derived from the classical Fourier integral. Based on the mathematical importance of the Aboodh Transform was first introduced in 2013 by Khalid Sulaiman Aboodh [14] to make the process of solving differential equations in time domain a bite easier. Aboodh Transform has a deeply connected with Laplace and Elzaki Transform [15]. This method is a useful technique for solving both linear and nonlinear differential equations. The major aim of this paper is to consider the applicability and effectiveness of the Aboodh Homotopy Perturbation method in solving Burgers equation. This techniques provides the solution in a rapidly convergent sequence with elegantly computed terms which leads the solution in a closed form. Its rapid convergence shows that the method is trustworthy and introduces a significant advancement in solving Burgers equation.

\section{ABOODH HOMOTOPY PERTURBATION METHOD}

The basic idea of this method can be illustrated by considering the general nonlinear homogeneous partial differential equation with initial conditions of the form [16]

$$
\begin{aligned}
& D U(x, t)+R U(x, t)+N U(x, t)=g(x, t) \\
& (x, 0)=h(x), U_{t}=(x, 0)=f(x)
\end{aligned}
$$

Where $D$ the second order linear differential operator is $D=\frac{\partial^{2}}{\partial t^{2}}, R$ is the linear differential operator of less order than $D, N$ represent the general nonlinear differential operator and $g(x, t)$ is the source term. 
Applying Aboodh Transform on both sides of equation (2.1),

$$
A[D U(x, t)+R U(x, t)+N U(x, t)]=A[g(x, t)]
$$

Applying the linearity property of the Aboodh Transform on equation (2.3), we have

$A[D U(x, t)]+[R U(x, t)]+N U(x, t)=g(x, t)$

$v^{2} U(x, v)-U(x, 0)-\frac{1}{v} U_{t}(x, 0)+A[R U(\dot{x}, t)]+A[N U(x, t)]=A[g(x, t)]$

Substituting the initial conditions in equation (2.2) into equation (2.5), we obtain

$v^{2} U(x, v)-h(x)-\frac{1}{v} f^{\prime}(x)=A[g(x, t)]-A[R U(x, t)]-A[N U(x, t)]$

$U(x, v)=\frac{1}{v^{2}} h(x)+\frac{1}{v^{3}} f^{\prime}(x)+\frac{1}{v^{2}} A[g(x, t)]-\frac{1}{v^{2}} A[R U(x, t)]-\frac{1}{v^{2}} A[N U(x, t)]$

$U(x, v)=\frac{1}{v^{2}} h(x)+\frac{1}{v^{3}} f(x)+\frac{1}{v^{2}} A[g(x, t)]-\frac{1}{v^{2}} A[R U(x, t)]-\frac{1}{v^{2}} A[N U(x, t)]$

Taking Aboodh inverse on both sides of equation (2.6), we obtain

$$
\begin{aligned}
& U(x, t)=A^{-1}\left[\frac{1}{v^{2}} h(x)+\frac{1}{v^{3}} f^{\prime}(x)+\frac{1}{v^{2}} A[g(x, t)]\right]-A^{-1} \frac{A}{v^{2}}[[R U(x, t)]-[N U(x, t)]] \\
& G(x, t)=A^{-1}\left[\frac{1}{v^{2}} h(x)+\frac{1}{v^{3}} f^{\prime}(x)+\frac{1}{v^{2}} A[g(x, t)]\right] \\
& U(x, t)=G(x, t)-A^{-1} \frac{A}{v^{2}}[[R U(x, t)]-[N U(x, t)]]
\end{aligned}
$$

Where $G(x, t)$ represent the terms arising from the source term and the prescribed initial conditions.

Assuming the solution of equation (2.1) is of the form

$U=U_{0}+p U_{1}+p^{2} U_{2}+p^{3} U_{3}+p^{4} U_{4}+\cdots$

To consider the nonlinear operator, we apply the homotopy perturbation method.

$U(x, t)=\sum_{n=0}^{\infty} p^{n} U_{n}(x, t)$

By substituting equation (2.11) into (2.9), we have

$$
\sum_{n=0}^{\infty} p^{n} U_{n}(x, t)=G(x, t)-p A^{-1}\left\{\frac{A}{v^{2}}\left[\left[R \sum_{n=0}^{\infty} p^{n} H_{n}(x, t)\right]-\left[N \sum_{n=0}^{\infty} p^{n} H_{n}(x, t)\right]\right]\right\}
$$

This is the coupling of the Aboodh Transform and the homotopy perturbation method. Comparing the coefficients of like powers of $p$, we have

$$
\begin{array}{ll}
p^{0}: & U_{0}(x, t)=G(x, t) \\
p^{1}: & U_{1}(x, t)=-A^{-1}\left\{\frac{A}{v^{2}}\left[\left[R U_{0}(x, t)\right]-\left[N U_{0}(x, t)\right]\right]\right\} \\
p^{2}: & U_{2}(x, t)=-A^{-1}\left\{\frac{A}{v^{2}}\left[\left[R U_{1}(x, t)\right]-\left[N U_{1}(x, t)\right]\right]\right\} \\
p^{3}: & U_{3}(x, t)=-A^{-1}\left\{\frac{A}{v^{2}}\left[\left[R U_{2}(x, t)\right]-\left[N U_{2}(x, t)\right]\right]\right\} \\
p^{4}: & U_{4}(x, t)=-A^{-1}\left\{\frac{A}{v^{2}}\left[\left[R U_{3}(x, t)\right]-\left[N U_{3}(x, t)\right]\right]\right\} \\
& \vdots
\end{array}
$$

In general, the recursive relation is given by:

$p^{m}: \quad U_{m}(x, t)=-A^{-1}\left\{\frac{A}{v^{2}}\left[\left[R U_{m}(x, t)\right]-\left[N U_{m}(x, t)\right]\right]\right\}$ 
Then, the solution can be expressed as

$U(x, t)=U_{0}(x, t)+U_{1}(x, t)+U_{2}(x, t)+\cdots$

\section{APPLICATION}

In this section, the Aboodh Homotopy perturbation method is implemented for solving Burgers equation with initial conditions. We demonstrate the applicability and the effectiveness of this method with three (3) numerical examples. The results obtained by this proposed method are compared with other known results.

\section{Example1}

Consider the following one dimensional Burgers equation as [16]

$U_{t}=U_{x x}-U U_{x}$

With initial conditions

$$
U(x, 0)=1-\frac{2}{x}
$$

Applying Aboodh Transform to equation (3.1)

$$
A\left[U_{t}\right]=A\left[U_{x x}-U U_{x}\right]
$$

Applying the linearity properties of Aboodh transform

$$
\begin{gathered}
A\left[U_{t}\right]=A\left[U_{x x}\right]-A\left[U U_{x}\right] \\
v U(x, v)-\frac{1}{v} U(x, 0)=A\left[U_{x x}\right]-A\left[U U_{x}\right]
\end{gathered}
$$

Substituting the initial conditions(3.2) into equation (3.4) we have

$$
\begin{array}{r}
v U(x, v)-\frac{1}{v}\left(1-\frac{2}{x}\right)=A\left[U_{x x}\right]-A\left[U U_{x}\right] \\
v U(x, v)=\frac{1}{v^{2}}\left(1-\frac{2}{x}\right)+\frac{A}{v}\left[U_{x x}\right]-\frac{A}{v}\left[U U_{x}\right]
\end{array}
$$

Now taking the Aboodh inverse on both sides of equation (3.5), we obtain

$$
\begin{array}{r}
U(x, t)=A^{-1}\left\{\frac{1}{v^{2}}\left(1-\frac{2}{x}\right)+\frac{A}{v}\left[U_{x x}\right]-\frac{A}{v}\left[U U_{x}\right]\right\} \\
U(x, t)=\left(1-\frac{2}{x}\right)+A^{-1}\left\{\frac{A}{v}\left[U_{x x}\right]-\frac{A}{v}\left[U U_{x}\right]\right\}
\end{array}
$$

Now, applying homotopy perturbation method,

$U(x, t)=\sum_{n=0}^{\infty} p^{n} U_{n}(x, t)$

Substituting equation (3.7) into (3.6)

$\sum_{n=0}^{\infty} p^{n} U_{n}(x, t)=\left(1-\frac{2}{x}\right)+p A^{-1}\left\{\frac{A}{v}\left\{\left(\sum_{n=0}^{\infty} p^{n} U_{n}(x, t)\right)_{x x}\right\}-\frac{A}{v}\left\{\left(\sum_{n=0}^{\infty} p^{n} U_{n} U_{n}(x, t)\right)_{x}\right\}\right\}$

Comparing the coefficients of the corresponding powers of $p$ in equation (3.8), we have

$$
\begin{aligned}
p^{0}: \quad U_{0}(x, t) & =1-\frac{2}{x} \\
p^{1} \vdots \quad U_{1}(x, t)= & A^{-1}\left\{\frac{A}{v}\left\{U_{0 x x}(x, t)\right\}-\frac{A}{v}\left\{U_{0}(x, t) U_{0 x}(x, t)\right\}\right\} \\
= & A^{-1}\left\{\frac{A}{v}\left(\frac{-4}{x^{3}}\right)-\frac{A}{v}\left(1-\frac{2}{x}\right)\left(\frac{2}{x^{2}}\right)\right\} \\
& =A^{-1}\left\{\frac{-4}{v^{3} x^{3}}-\frac{4}{v^{3} x^{2}}+\frac{4}{v^{3} x^{3}}\right\}
\end{aligned}
$$




$$
\begin{aligned}
& U_{1}(x, t)=-\frac{2}{x^{2}} t \\
& p^{2}: \quad U_{2}(x, t)=A^{-1}\left\{\frac{A}{v}\left\{U_{1 x x}(x, t)\right\}-\frac{A}{v}\left[\left\{U_{1} U_{0 x}\right\}+U_{0} U_{1 x}\right]\right\} \\
& U_{2}(x, t)=A^{-1}\left\{\frac{A}{v}\left[\frac{-12}{x^{4}} t\right]-\frac{A}{v}\left[\left(\frac{-2}{x^{2}} t\right)\left(\frac{2}{x^{2}}\right)+\left(1-\frac{2}{x}\right)\left(\frac{4}{x^{3}} t\right)\right]\right\} \\
& U_{2}(x, t)=A^{-1}\left\{\frac{A}{v}\left[-\frac{12}{x^{4}} t+\frac{12}{x^{4}} t-\frac{4}{x^{3}} t\right]\right\} \\
& U_{2}(x, t)=\frac{-2}{x^{3}} t^{2} \\
& p^{3}: \quad U_{3}(x, t)=A^{-1}\left\{\frac{A}{v}\left\{U_{2 x x}(x, t)\right\}-\frac{A}{v}\left[U_{2} U_{0 x}+U_{1} U_{1 x}+U_{0} U_{2 x}\right]\right\} \\
& U_{3}(x, t)=A^{-1}\left\{\frac{A}{v}\left[\frac{-24}{x^{5}} t^{2}\right]-\frac{A}{v}\left[-\frac{12}{x^{5}} t^{2}+\frac{6}{x^{4}} t^{2}-\frac{12}{x^{5}} t^{2}\right]\right\} \\
& U_{3}(x, t)=-\frac{2}{x^{4}} t^{3} \\
& p^{4}: \quad U_{4}(x, t)=A^{-1}\left\{\frac{A}{v}\left\{U_{3 x x}(x, t)\right\}-\frac{A}{v}\left[\left\{U_{3} U_{0 x}\right\}+U_{2} U_{1 x}+U_{1} U_{2 x}+U_{0} U_{3 x}\right]\right\} \\
& U_{4}(x, t)=A^{-1}\left\{\frac{A}{v}\left[\frac{-40}{x^{6}} t^{3}\right]-\frac{A}{v}\left[-\frac{24}{x^{6}} t^{3}+\frac{8}{x^{5}} t^{3}-\frac{16}{x^{6}} t^{3}\right]\right\} \\
& U_{4}(x, t)=A^{-1}\left\{\frac{-8}{x^{5}} t^{3}\right\} \\
& U_{4}(x, t)=\frac{-2}{x^{5}} t^{4}
\end{aligned}
$$

Then, the solution $U(x, t)$ is expressed as

$$
\begin{array}{r}
U(x, t)=U_{0}(x, t)+U_{1}(x, t)+U_{2}(x, t)+U_{3}(x, t)+U_{4}(x, t)+\cdots \\
U(x, t)=\left(1-\frac{2}{x}\right)+\left(\frac{-2}{x^{2}} t\right)+\left(\frac{-2}{x^{3}} t^{2}\right)+\left(-\frac{2}{x^{4}} t^{3}\right)+\left(\frac{-2}{x^{5}} t^{4}\right) \\
U(x, t)=1-\frac{2}{x}-\frac{2}{x^{2}} t-\frac{2}{x^{3}} t^{2}-\frac{2}{x^{4}} t^{3}-\frac{2}{x^{5}} t^{4}
\end{array}
$$

Thus, the solution can be written in the closed form as:

$$
U(x, t)=1-\frac{2}{(x-t)}
$$

Equation (3.10) is the exact solution for equation (3.1) which is the same as the solution in [16]

\section{Example 2}

Consider one-dimensional Burger's equation of the form [16]

$U_{t}=U_{x x}-U U_{x}$

Subject to initial condition

$$
U(x, 0)=x
$$

Applying Aboodh Transform to equation (3.11)

$$
A\left[U_{t}\right]=A\left[U_{x x}-U U_{x}\right]
$$

Applying the linearity properties of Aboodh transform 


$$
\begin{gathered}
A\left[U_{t}\right]=A\left[U_{x x}\right]-A\left[U U_{x}\right] \\
v U(x, v)-\frac{1}{v} U(x, 0)=A\left[U_{x x}\right]-A\left[U U_{x}\right]
\end{gathered}
$$

Substituting the initial conditions(3.12) into equation (3.14) we have

$$
\begin{array}{r}
v U(x, v)-\frac{x}{v}=A\left[U_{x x}\right]-A\left[U U_{x}\right] \\
v U(x, v)=\frac{x}{v^{2}}+\frac{A}{v}\left[U_{x x}\right]-\frac{A}{v}\left[U U_{x}\right]
\end{array}
$$

Now taking the Aboodh inverse on both sides of equation (3.15), we obtain

$$
\begin{aligned}
& U(x, t)=A^{-1}\left\{\frac{x}{v^{2}}+\frac{A}{v}\left[U_{x x}\right]-\frac{A}{v}\left[U U_{x}\right]\right\} \\
& U(x, t)=x+A^{-1}\left\{\frac{A}{v}\left[U_{x x}\right]-\frac{A}{v}\left[U U_{x}\right]\right\}
\end{aligned}
$$

Now, applying Homotopy perturbation method

$U(x, t)=\sum_{n=0}^{\infty} p^{n} U_{n}(x, t)$

Substituting equation (3.17) into (3.16)

$\sum_{n=0}^{\infty} p^{n} U_{n}(x, t)=x+p A^{-1}\left\{\frac{A}{v}\left\{\left(\sum_{n=0}^{\infty} p^{n} U_{n}(x, t)\right)_{x x}\right\}-\frac{A}{v}\left\{\left(\sum_{n=0}^{\infty} p^{n} U_{n} U_{n}(x, t)\right)_{x}\right\}\right\}$

Comparing the coefficients of the corresponding powers of $p$ in equation (3.18), we obtain

$$
\begin{gathered}
p^{0}: \quad U_{0}(x, t)=x \\
p^{1}: \quad U_{1}(x, t)=A^{-1}\left\{\frac{A}{v}\left\{U_{0 x x}(x, t)\right\}-\frac{A}{v}\left\{U_{0}(x, t) U_{0 x}(x, t)\right\}\right\} \\
U_{1}(x, t)=A^{-1}\left\{\frac{A}{v}\{0\}-\frac{A}{v}\{x\}\right\} \\
U_{1}(x, t)=-x t \\
p^{2}: \quad U_{2}(x, t)=A^{-1}\left\{\frac{A}{v}\left\{U_{1 x x}(x, t)\right\}-\frac{A}{v}\left[\left\{U_{1} U_{0 x}\right\}+U_{0} U_{1 x}\right]\right\} \\
U_{2}(x, t)=A^{-1}\left\{\frac{A}{v}[0]-\frac{A}{v}[(-x t)(1)+(x)(-t)]\right\} \\
U_{2}(x, t)=-A^{-1}\left\{\frac{A}{v}[-2 x t]\right\} \\
U_{2}(x, t)=x t^{2} \\
p^{3} \vdots \quad U_{3}(x, t)=A^{-1}\left\{\frac{A}{v}\left\{U_{2 x x}(x, t)\right\}-\frac{A}{v}\left[\left\{U_{2} U_{0 x}\right\}+U_{1} U_{1 x}+U_{0} U_{2 x}\right]\right\} \\
U_{3}(x, t)=A^{-1}\left\{\frac{A}{v}[0]-\frac{A}{v}\left[x t^{2}+2 x t^{2}+x t^{2}\right]\right\} \\
U_{3}(x, t)=-A^{-1}\left\{\frac{A}{v}\left[3 x t^{2}\right]\right\} \\
U_{3}(x, t)=-x t^{3} \\
p^{4} \vdots \quad U_{4}(x, t)=A^{-1}\left\{\frac{A}{v}\left\{U_{3 x x}(x, t)\right\}-\frac{A}{v}\left[\left\{U_{3} U_{0 x}\right\}+U_{2} U_{1 x}+U_{1} U_{2 x}+U_{0} U_{3 x}\right]\right\}
\end{gathered}
$$




$$
\begin{gathered}
U_{4}(x, t)=A^{-1}\left\{\frac{A}{v}[0]-\frac{A}{v}\left[-x t^{3}-x t^{3}-x t^{3}-x t^{3}\right]\right\} \\
U_{4}(x, t)=A^{-1}\left\{\frac{A}{v}\left[4 x t^{3}\right]\right\} \\
U_{4}(x, t)=x t^{4}
\end{gathered}
$$

Then, the solution $U(x, t)$ is expressed as

$$
U(x, t)=U_{0}(x, t)+U_{1}(x, t)+U_{2}(x, t)+U_{3}(x, t)+U_{4}(x, t)+\cdots
$$

$U(x, t)=x-x t+x t^{2}-x t^{3}+x t^{4}-\cdots$

$U(x, t)=x\left(-t+t^{2}-t^{3}+t^{4}-\cdots\right)$

Thus, the solution can be written in the closed form as:

$U(x, t)=\frac{x}{1+t}$

Equation (3.21) is in good agreement with equation (3.11) and (3.12). It can be verified by direct substitution.

\section{Example 3}

Consider one-dimensional Burger's equation of the form [17]

$U_{t}+U U_{x}=U_{x x}$

Subject to initial condition

$$
U(x, 0)=2 x
$$

Applying Aboodh Transform to equation (3.21)

$$
A\left[U_{t}+U U_{x}\right]=A\left[U_{x x}\right]
$$

Applying the linearity properties of Aboodh transform

$$
\begin{gathered}
A\left[U_{t}\right]+A\left[U U_{x}\right]=A\left[U_{x x}\right] \\
v U(x, v)-\frac{1}{v} U(x, 0)+A\left[U U_{x}\right]=A\left[U_{x x}\right]
\end{gathered}
$$

Substituting the initial conditions(3.22) into equation (3.24) we have

$$
\begin{array}{r}
v U(x, v)-\frac{2 x}{v}=A\left[U_{x x}\right]-A\left[U U_{x}\right] \\
v U(x, v)=\frac{2 x}{v^{2}}+\frac{A}{v}\left[U_{x x}\right]-\frac{A}{v}\left[U U_{x}\right]
\end{array}
$$

Now, taking the Aboodh inverse on both sides of equation (3.25), we obtain

$$
\begin{aligned}
& U(x, t)=A^{-1}\left\{\frac{2 x}{v^{2}}+\frac{A}{v}\left[U_{x x}\right]-\frac{A}{v}\left[U U_{x}\right]\right\} \\
& U(x, t)=2 x+A^{-1}\left\{\frac{A}{v}\left[U_{x x}\right]-\frac{A}{v}\left[U U_{x}\right]\right\}
\end{aligned}
$$

Now, applying Homotopy perturbation method, we assume that

$U(x, t)=\sum_{n=0}^{\infty} p^{n} U_{n}(x, t)$

Substituting equation (3.27) into (3.26)

$\sum_{n=0}^{\infty} p^{n} U_{n}(x, t)=2 x+p A^{-1}\left\{\frac{A}{v}\left\{\left(\sum_{n=0}^{\infty} p^{n} U_{n}(x, t)\right)_{x x}\right\}-\frac{A}{v}\left\{\left(\sum_{n=0}^{\infty} p^{n} U_{n} U_{n}(x, t)\right)_{x}\right\}\right\}$

Comparing the coefficients of the corresponding powers of $p$ in equation (3.28), we obtain 
$p^{0}: \quad U_{0}(x, t)=2 x$

$p^{1}: \quad U_{1}(x, t)=A^{-1}\left\{\frac{A}{v}\left\{U_{0 x x}(x, t)\right\}-\frac{A}{v}\left\{U_{0}(x, t) U_{0 x}(x, t)\right\}\right\}$

$$
\begin{gathered}
U_{1}(x, t)=A^{-1}\left\{\frac{A}{v}\{0\}-\frac{A}{v}\{4 x\}\right\} \\
U_{1}(x, t)=-4 x t
\end{gathered}
$$

$p^{2}: \quad U_{2}(x, t)=A^{-1}\left\{\frac{A}{v}\left\{U_{1 x x}(x, t)\right\}-\frac{A}{v}\left[\left\{U_{1} U_{0 x}\right\}+U_{0} U_{1 x}\right]\right\}$

$$
\begin{gathered}
U_{2}(x, t)=A^{-1}\left\{\frac{A}{v}[0]-\frac{A}{v}[(-4 x t)(2)+(2 x)(-4 t)]\right\} \\
U_{2}(x, t)=-A^{-1}\left\{\frac{A}{v}[-16 x t]\right\} \\
U_{2}(x, t)=8 x t^{2}
\end{gathered}
$$

$$
\begin{gathered}
p^{3}: \quad U_{3}(x, t)=A^{-1}\left\{\frac{A}{v}\left\{U_{2 x x}(x, t)\right\}-\frac{A}{v}\left[\left\{U_{2} U_{0 x}\right\}+U_{1} U_{1 x}+U_{0} U_{2 x}\right]\right\} \\
U_{3}(x, t)=A^{-1}\left\{\frac{A}{v}[0]-\frac{A}{v}\left[16 x t^{2}+16 x t^{2}+16 x t^{2}\right]\right\} \\
U_{3}(x, t)=-A^{-1}\left\{\frac{A}{v}\left[48 x t^{2}\right]\right\} \\
U_{3}(x, t)=-16 x t^{3}
\end{gathered}
$$

$p^{4}: \quad U_{4}(x, t)=A^{-1}\left\{\frac{A}{v}\left\{U_{3 x x}(x, t)\right\}-\frac{A}{v}\left[U_{3} U_{0 x}+U_{2} U_{1 x}+U_{1} U_{2 x}+U_{0} U_{3 x}\right]\right\}$

$$
\begin{gathered}
U_{4}(x, t)=A^{-1}\left\{\frac{A}{v}[0]-\frac{A}{v}\left[-32 x t^{3}-32 x t^{3}-32 x t^{3}-32 x t^{3}\right]\right\} \\
U_{4}(x, t)=A^{-1}\left\{\frac{A}{v}\left[128 x t^{3}\right]\right\} \\
U_{4}(x, t)=32 x t^{4}
\end{gathered}
$$

$p^{5}: \quad U_{5}(x, t)=A^{-1}\left\{\frac{A}{v}\left\{U_{4 x x}(x, t)\right\}-\frac{A}{v}\left[U_{4} U_{0 x}+U_{3} U_{1 x}+U_{2} U_{2 x}+U_{1} U_{3 x}+U_{0} U_{4 x}\right]\right\}$

$$
\begin{gathered}
U_{4}(x, t)=A^{-1}\left\{\frac{A}{v}[0]-\frac{A}{v}\left[64 x t^{4}+64 x t^{4}+64 x t^{4}+64 x t^{4}+64 x t^{4}\right]\right\} \\
U_{4}(x, t)=A^{-1}\left\{\frac{A}{v}\left[320 x t^{4}\right]\right\} \\
U_{4}(x, t)=A^{-1}\left\{\frac{7680}{v^{7}}\right\} \\
U_{4}(x, t)=-64 x t^{5}
\end{gathered}
$$

Then, the solution $U(x, t)$ is expressed as

$$
\begin{aligned}
& \quad U(x, t)=U_{0}(x, t)+U_{1}(x, t)+U_{2}(x, t)+U_{3}(x, t)+U_{4}(x, t)+U_{4}(x, t)+\cdots \\
& U(x, t)=2 x-4 x t+8 x t^{2}-16 x t^{3}+32 x t^{4}-64 x t^{5}+\cdots \\
& U(x, t)=2 x\left(1-2 t+4 t^{2}-8 t^{3}+16 t^{4}-32 t^{5}+\cdots\right)
\end{aligned}
$$


Thus, the solution can be written in the closed form as:

$U(x, t)=\frac{2 x}{1+2 t}$

Equation (3.31) is the same as the result obtained in [17]

\section{CONCLUSION}

In this paper, we have introduced the Aboodh homotopy perturbation method for the solution of Burgers equations. The main advantage of this method is that the method is applied in a direct way without any linearization or discretization. From the obtained results, we conclude that the Aboodh Homotopy Perturbation method is an extremely powerful and efficient method in providing analytical solutions for nonlinear problems and can be used in obtaining the exact solution in a rapidly convergent sequence with elegantly computed terms. Its rapid convergence shows that the method is trustworthy and introduces a significant advancement in solving Burgers equation. This method can also be applied to provide solutions for diverse classes of nonlinear problems.

\section{REFERENCES}

[1] Rogers, C. and Shadwich, W. F. Backlund Transformations and Their applications, New York: Academic Press, 1982.

[2] Hirota, R.” Exact solutions of the Korteweg-De vries Equation for Multiple collisions of Solitons". Physical Review Letters, vol. 27, no. 18, pp. 1192-1194, 1971.

[3] He, J. H. "Variational iteration method for autonomous ordinary differential systems" pp. 115-123, 2000.

[4] He, J. H. "Approximate Analytical solution for seepage flow with fractional derivatives in porous media", Computer Methods in Applied Mechanics and Engineering, vol.167, no.1-2, pp. 57-68, 1998.

[5] Adomian, G. "A Review of the Decomposition Method in Applied Mathematics", Journal of Mathematical Analysis and Applications, 135, 501-544, 1988.

[6] Liao, S. J. "The proposed homotopy analysis technique for the solution of nonlinear problems", PhD Thesis, Shanghai Jiao Tong University, 1992.

[7] Liao, S. J. “An explicit, totally analytic approximate solution”, International Journal of Non-Linear Mechanics, vol. 34, pp. 759-778, 1999 .

[8] Liao, S. J. "On the homotopy analysis method for nonlinear problems", Journal of Applied Mathematics and Computation, vol. 147, no. 2, pp. 499-513, 2004

[9] Liao, S. J. "Series solutions of unsteady boundary-layer flows over a stretching flat plate", Studies in Applied Mathematics, vol. 117, no. 3, pp.239-263, 2006.

[10] He, J. H. "The homotopy perturbation method for nonlinear oscillators with discontinuities" Journal of Applied Mathematics and computation, vol.151, no. 1, pp. 287-292, 2004.

[11] He, J. H. “Application of homotopy perturbation method”, Journal of Chaos, Solitons and Fractals, vol. 26, pp. 695-700, 2005.

[12] He, J. H. "Homotopy perturbation method: a new nonlinear analytical technique", Journal of Applied Mathematics and Computations, pp. 73-79, 2003.

[13] He, J. H. "A coupling method of a homotopy technique and a perturbation technique for non-linear problems", International Journal of Non-Linear Mechanics, vol. 35, pp. 37-43, 2000.

[14] Aboodh, K. S. "The New Integral Transform Aboodh Transform", Global Journal of Pure and Applied Mathematics ISSN0973-1768, vol. 9, no. 1, pp. 35-43, 2013.

[15] Aboodh, K. S. “Application of New "Aboodh Transform" to Partial Differential Equations”, Global Journal of pure and Applied Mathematics, vol. 10, no. 2, pp. 249-254, 2014.

[16] Ahamad J, Bibi Z, Noor K. "Laplace Decomposition Method using He's Polynomial to Burgers Equation" Journal of Science and Arts, vol. 2, no. 27, pp. 131-138, 2014.

[17] Biazar, J. and Ghazvini, H. "Exact Solutions for nonlinear Burgers equation by Homotopy Perturbation method", Journal of Numerical Methods for Partial Differential Equations, 2008. 